\title{
Comparison of OFDM and OOK Modulations for Vehicle-to-Vehicle Visible Light Communication in Real-World Driving Scenarios
}

\author{
Bastien Béchadergue ${ }^{\mathrm{a}, \mathrm{b}}$, Wen-Hsuan Shen ${ }^{\mathrm{c}}$, Hsin-Mu Tsai ${ }^{\mathrm{c}, *}$ \\ ${ }^{a}$ Vedecom Insitute, 77 rue des Chantiers, 78000 Versailles, France \\ ${ }^{b}$ Laboratoire d'Ingénierie des Systèmes de Versailles (LISV), UVSQ, Université Paris-Saclay, 10-12 avenue de l'Europe, 78140, Vélizy, France \\ ${ }^{c}$ Department of Computer Science and Information Engineering, National Taiwan University, Taipei 10617, Taiwan
}

\begin{abstract}
Visible light communication (VLC) is seen as an interesting technology to complement the IEEE 802.11p-based systems commonly used for vehicle-to-vehicle (V2V) communication. However, the reliability of such a V2V-VLC link in real-world driving scenario had not been demonstrated up to very recently. The results obtained, at that time with orthogonal frequency division multiplexing (OFDM) at $2 \mathrm{kbps}$, are complemented in this paper with additional results using on-off keying (OOK) at $100 \mathrm{kbps}$. The overall performances of this second modulation are rather close to those of OFDM. The packet reception rate (PRR) remains more than $90 \%$ over a service area of $30 \mathrm{~m}$ length-wise and the link is not affected by multipath propagation. Error-free transmission is even demonstrated over $60 \mathrm{~m}$ using repetition coding, at the cost of a reduced data rate of $10 \mathrm{kbps}$. However, it is shown that OFDM is able to cope with the narrow-band interferences generated by outdoor lighting such as LED signs whereas the OOK link is completely jammed. Despite its simplicity and good overall performances, OOK is thus not as robust as OFDM to the variety of situations experienced in real-world driving scenario.
\end{abstract}

Keywords: visible light communication, vehicular communication, OFDM, OOK

PACS: [2010] 42.79.Sz.

\section{Introduction}

Vehicular communication is a cornerstone of future autonomous vehicles and is thus progressively being introduced in the latest vehicles to enable new cooperative driving functions. Using IEEE 802.11p-based wireless communication technologies such as dedicated short range communication (DSRC) or cooperative intelligent transportation system (C-ITS), vehicular communication is possible over a few hundreds of meters with a theoretical data rate of $27 \mathrm{Mbps}$ [1]. However, these technologies are so sensitive to interferences that the medium access control (MAC) layer described in the IEEE $802.11 \mathrm{p}$ standard defines heavy protocols to address the potential channel congestion issues that could appear, especially in dense traffic scenario. These protocols induce eventually an increased transmission latency that might not be compatible with the most safety critical applications [2], such as automated platoons of vehicles. Such platoons rely on a very small V2V distance in

${ }^{*}$ Corresponding author

Preprint submitted to Ad Hoc Networks order to increase traffic flow and reduce fuel consumption [3]. For example, the European Truck Platooning network targets a $0.3 \mathrm{~s}$ V2V gap on highways, which corresponds to $10.8 \mathrm{~m}$ at $130 \mathrm{~km} / \mathrm{h}$ [4]. In this case, the following vehicles must react immediately, hence the needs of very low latency V2V communication.

Recently, visible light communication (VLC) has emerged as an alternative technology for vehicular communication, by using light-emitting diode (LED) headlamps or taillights as transmitters and photodiodes (PD) or cameras as receivers. The resulting optical signals are highly directional and propagate primarily with line-ofsight paths, which leads to reduced channel congestion. Therefore, VLC is considered to have lower sensitivity to interferences, which enables point-to-point transmission of data with very light protocols and low latency even under high vehicle density scenarios. Several works have already implemented VLC systems for automotive applications [5]. In [6], an infrastructure-tovehicle (I2V) system is implemented using a sophisticated high-speed camera receiver. Error-free communication at $16 \mathrm{kbps}$ is demonstrated while the vehicle is

July 18, 2019 
driving toward the traffic light from $80 \mathrm{~m}$ at $30 \mathrm{~km} / \mathrm{h}$. The data rate has then been brought up to $3.1 \mathrm{Mbps}$ using a PD receiver with orthogonal frequency division multiplexing (OFDM) for the application of universal traffic management system in Japan [7]. In [8], a vehicle-to-vehicle (V2V) communication system enabling a 10 Mbps link between two following vehicles is demonstrated. However, the transmitting lights are custom-made matrices of LED and the receiver is a highly-customized non-commercial image sensor.

The development of VLC for automotive application suffers from the lack of a realistic and easy-toimplement proof-of-concept in real driving situations. Such a proof-of-concept has only been realized very recently, in our previous work [9], where a one-way link between two adjacent vehicles is established. The taillights of the leading vehicle (LV) are driven with an OFDM modulation whereas the following vehicle (FV) is equipped with a simple PD on its front bumper. A robust communication channel is demonstrated in realworld driving scenarios on highways over $35 \mathrm{~m}$ lengthwise and with $\pm 4 \mathrm{~m}$ lateral shifts between the vehicles. However, even though very light communication protocols are used, the data rate of $2 \mathrm{kbps}$ reached in this work limits the transmission latency. On the other hand, indoor tests showed that a latency of $4.2 \mathrm{~ms}$ could be reached when transmitting 400 bits packets that contain the most vital data of the vehicle at $100 \mathrm{kbps}$, using the very standard on-off keying (OOK) transmission scheme with Manchester coding [10].

Consequently, in this work, we replace the OFDM modulation used in [9] with OOK with Manchester coding, to compare its reliability with OFDM in real-world driving scenarios. We first present the reception rate performance of both modulation schemes when used with the same output light power. While using OOK, the packet reception rate (PRR) over 625 thousand packets (i.e., more than 250 millions bits) remains larger than $90 \%$ over $30 \mathrm{~m}$ despite lateral shifts of $\pm 1 \mathrm{~m}$ and is not affected at all by inter-symbol interferences (ISI). This range is even extended to $60 \mathrm{~m}$ when using a repetition coding scheme that however limited the data rate to 10 kbps. The obtained results present similar performances as when using OFDM. The robustness of both modulations to interferences caused by road environments is then compared. Strong external narrow-band interferences coming from LED signs are observed. It is shown that OFDM is less impacted than OOK by these interferences, thanks to the data parallelization over several orthogonal subcarriers. Therefore, even though OOK exhibits interesting simplicity and range characteristics, OFDM appears to be more robust to the variety of con- figurations experienced in real-world driving situations. These results are further detailed in Section 3, after presenting the prototype implementation in Section 2 and before concluding this paper with additional remarks and relevant future works in Section 4.

\section{System Design and Implementation}

The experiments carried out in this work are based on the same prototype as in [9], while only the modulation scheme has been changed for OOK with Manchester coding. Therefore, in this section, the motivations that lead to this choice are first exposed with all the details concerning its implementation. The benefits and parameters of the OFDM scheme previously used are then recalled, along with the overall experimental setup.

\subsection{Modulation Formats}

\subsubsection{A Single Carrier Modulation, $O O K$}

OOK is the most common single carrier modulation (SCM), where a data bit 1 is sent by turning the transmitting light on and a data bit 0 is sent by turning it off. Pulse position modulation (PPM) and pulse amplitude modulation (PAM) are two other typical SCM having their own advantages and drawbacks. For example, high-order PPM provides good power efficiency but reduced spectral efficiency. Conversely, PAM has an increasing spectral efficiency when its order gets larger but also a reduced power efficiency. OOK is thus an interesting compromise between PPM and PAM, especially for automotive applications. The modulation bandwidth of the LED headlamps and taillights is indeed limited to a few megahertz which means spectral efficiency is the most constraining parameter and thus excludes high-order PPM. On the other hand, the LED non-linearity limits the use of high-order PAM, as shown in [10].

Although OOK is an interesting candidate for our application, most of its spectral content is unfortunately gathered around the direct current (DC) level. Therefore, it is rather difficult to filter out the contribution of ambient lighting without cutting the data signal itself. To solve this issue, we add a simple Manchester coding which shifts the main lobe of the data signal spectrum around the data rate. The data bits are thus first Manchester-encoded and then sent at a clock rate $f_{c}=$ $200 \mathrm{kHz}$, which gives a data rate $R_{b}=100 \mathrm{kbps}$. In practice, this data is transmitted in packets of 400 bits preceded by the simple header $H=1111$, which leads to a transmission latency of $4.2 \mathrm{~ms}$ [10]. 



(c)

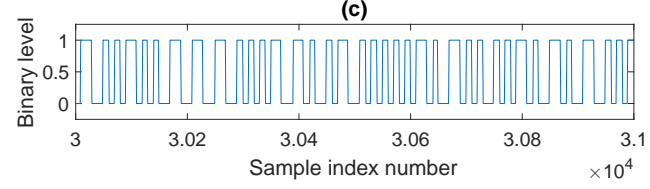

Figure 1: Manchester signal (a) received by the PD with a DC level, (b) after bandpass filtering and (c) after zero-crossing detection.

Once received, the data signal is first sampled at a frequency $f_{s}=2 \mathrm{MHz}$, then reconstructed by a signal processing stage and finally decoded. The reconstruction is made by a 2nd-order bandpass filter followed by a zero-crossing detection stage. By carefully choosing the high-pass and low-pass cut-off frequencies, the bandpass filter can cut the DC level of the signal received, and thus the ambient lighting contribution, while removing a large part of the receiver noise. As recommended in [11], the high-pass and low-pass cut-off frequencies were set respectively at $0.01 R_{b}$ and $R_{b}$, that is $1 \mathrm{kHz}$ and $100 \mathrm{kHz}$. As illustrated by Figure 1, this filtering stage centers the signal around the null level and thus allows zero-crossing detection to reconstruct the binary data signal that is finally decoded.

\subsubsection{A Multiple Carrier Modulation, OFDM}

Despite its benefits, OOK modulation with Manchester coding also suffers from limitations. First of all, if we want to increase the data rate, the only solution consists in rising the clock rate $f_{c}$ and thus reduces the symbol duration. In addition to the LED bandwidth limitation already evoked, other issues will appear in this case. In particular, at some point, typically from data rates around $10 \mathrm{Mbps}$, the symbol duration will fall below the delay spread of the channel. In other words, the system will become very sensitive to inter-symbol interferences (ISI), unless complex equalization techniques are used [11]. However, there is an even more crucial limitation. Since the data are contained on a single carrier, external interferences on this carrier might have dramatic impact of the transmission performances of the system as they cannot be filtered out without altering the data signal itself.

To overcome these issues, multiple carrier modulations (MCM) such as OFDM can be used. In OFDM, the data stream is parallelized and sent through orthogonal subcarriers. Each sub-stream can be modulated using a high-order modulation such as phase-shift keying (PSK) or quadrature amplitude modulation (QAM). This allows a high data rate while maintaining longer symbol duration than that of SCM which limits the ISI impact. Furthermore, external narrow-band interferences will most probably impact only a limited number of subcarriers while the other subcarriers will remain unaltered, which in turn leads to better error rate performances.

In [9], 16 subcarriers are used, which ensures a sufficient signal-to-noise ratio (SNR) per subcarrier for efficient decoding. However, only the central 14 subcarriers are effectively used, as the edge subcarriers are more likely to be affected by noises. Among these 14 subcarriers, 10 are used to convey data while 4 are dedicated to pilot symbol transmissions. In addition, a cyclic prefix of 4 samples is added as guard interval before each OFDM symbol in order to further limit ISI. The subcarriers are modulated at frequencies from $200 \mathrm{kHz}$ to $400 \mathrm{kHz}$ using binary PSK (BPSK) and generated with a digital-to-analog converter (DAC) at a sampling rate of $200 \mathrm{kHz}$. As the median of $90 \%$ coherence time of the V2V-VLC channel on the freeway is about $0.2 \mathrm{~s}$ [12], the packet duration is set to be $0.2 \mathrm{~s}$, corresponding to 40 symbols carrying 400 bits per packet. In addition, each OFDM symbol is repeated 50 times which, as shown in [9], enhances the decoding power, and thus, the range of operation. The repetition coding of 50 times is determined empirically based on the experimental results to satisfy our target range of $45 \mathrm{~m}$. For a larger target range, one can simply increase the number of repetitions. However, this repetition coding would limit the data rate, in this case, to $R_{b}=2 \mathrm{kbps}$. Finally, the decoding is performed digitally after sampling the received signal at a rate $f_{s}=200 \mathrm{kHz}$.

\subsection{Theoretical Comparison of the OOK and OFDM}

Both the performances of the OOK with Manchester coding and OFDM schemes strongly depend on the SNR of the signal received. This SNR depends itself on the VLC channel, generally composed of a line-ofsight (LOS) component and a non-LOS (NLOS) component. However, the NLOS component is negligible in most non-wet road surface scenarios [13]. Assuming a dry road, the received optical power by a vehicle $P_{r}$ is 


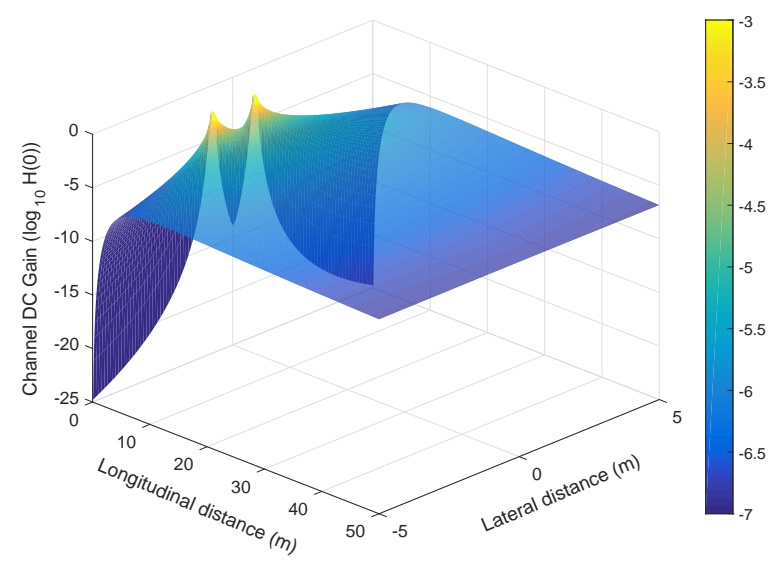

Figure 2: Spatial distribution of the VLC LOS channel DC gain $H(0)$ in the case of $\mathrm{V} 2 \mathrm{~V}$ communication. The transmitters are assumed to be located at the peaks of $H(0)$.

mostly equal to the LOS contribution of the transmitted optical power $P_{t}$, altered by the LOS channel. This channel is usually modeled as a path loss model along with an additive white Gaussian noise (AWGN) channel [11] with a frequency response $H(f)$ reduced to its DC gain $H(0)$ because it is considered flat in the frequency range of interest. Therefore, the received optical power $P_{r}$ is defined as:

$$
P_{r}=H(0) P_{t}
$$

The channel DC gain $H(0)$ then depends on the type of light source used, especially on its beam pattern. [14] shows that the Lambertian model is applicable when the vehicle bearing is less than 10 degrees, which is the case in our real-world experiments. Therefore, the DC gain is defined as:

$$
H(0)=\frac{(m+1) A_{r}}{2 \pi d^{2}} \cos ^{m} \phi \cos \psi, \quad 0<\psi<\psi_{c},
$$

where $\phi$ and $\psi$ are the irradiance and incidence angles, $A_{r}$ is the radiant sensitive area of the PD, $\psi_{c}$ is the PD field of view (FOV) and $m$ is the order of Lambertian emission, defined as $m=-\ln 2 / \ln \left(\cos \Phi_{1 / 2}\right)$, where $\Phi_{1 / 2}$ is the semi-angle at half power of the LED emitter. It appears clearly that $H(0)$ and thus $P_{t}$ are strongly impacted by the $\mathrm{V} 2 \mathrm{~V}$ absolute distance $d$ but also by the relative angles between the two vehicles. Fig. 2 illustrates this dependance by showing the spatial distribution of $H(0)$ when both taillights of a vehicle are emitting a VLC signal.

On the other hand, the SNR depends on the noise at the receiver level. In outdoor VLC, this noise is usually considered as a signal-independent Gaussian noise

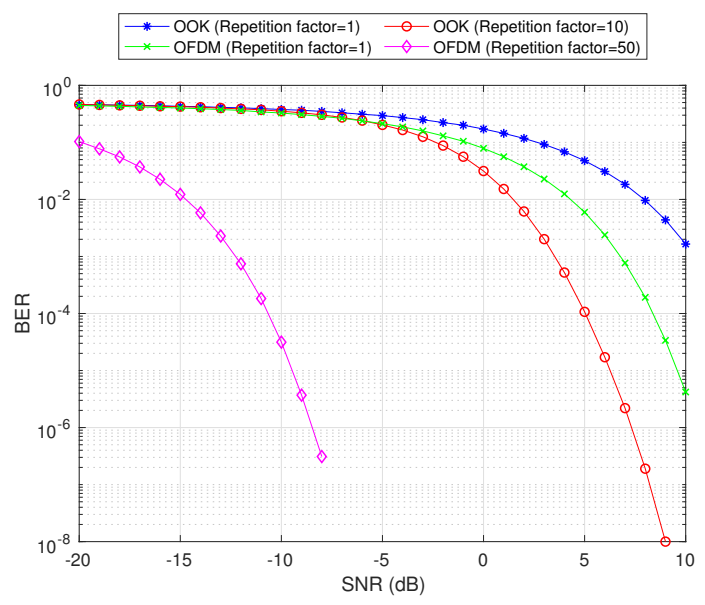

Figure 3: BER with respect to SNR comparison of OOK and OFDM.

resulting from the addition of shot noise, mainly due to daylight and thermal noise of respective variance $\sigma_{\text {shot }}^{2}$ and $\sigma_{\text {thermal }}^{2}$. Several closed-form expressions exist for these variances, depending on the photo-receiver technology [11]. In all cases, the SNR is eventually defined as:

$$
\mathrm{SNR}=\frac{S}{\sigma_{\text {tot }}^{2}}=\frac{\gamma^{2} H(0)^{2} P_{t}^{2}}{\sigma_{\text {shot }}^{2}+\sigma_{\text {thermal }}^{2}},
$$

where $\sigma_{\text {tot }}^{2}=\sigma_{\text {shot }}^{2}+\sigma_{\text {thermal }}^{2}$ is the variance of background noise and $\gamma$ is the responsivity of the PD.

By using Monte-Carlo simulations, the bit error rate (BER) evolution with the SNR can be evaluated. In order to provide a comparison to the real-world experiments that will be detailed in Section 3, we simulate the BER performance of OOK with Manchester coding and OFDM both with and without the repetition factor. Figure 3 shows the simulation results. In the case that repetition is applied to both modulation schemes, OFDM clearly has better BER performances than OOK with Manchester coding. This advantage partly comes from the larger repetition factor, but is also intrinsic since the BER of OFDM without repetition coding is still lower than the BER of OOK with Manchester coding without repetition coding. Consequently, we can expect OFDM to reach larger communication range than OOK. In parallel, we can also expect repetition to improve the communication range since it reduces the BER for any given SNR and for both the OFDM and OOK schemes.

\subsection{VLC System Implementation}

The two modulations just described are at the heart of the VLC system shown on Figure 4(a) that has been implemented on two 2015 Ford Focus C346 1.6L 5-doors, 


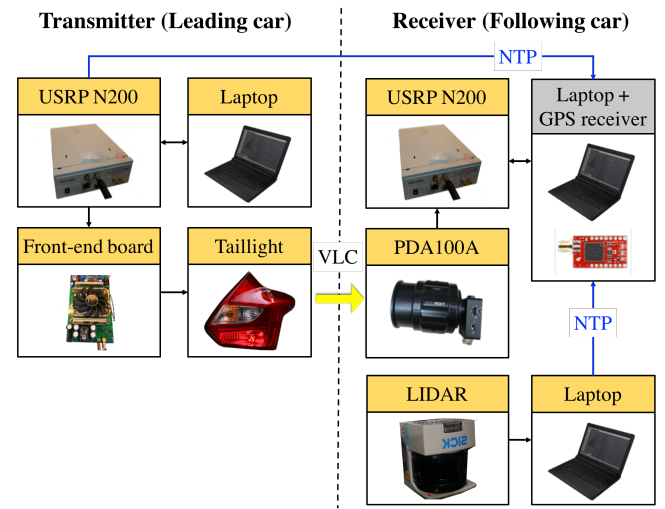

(a) Block diagram.

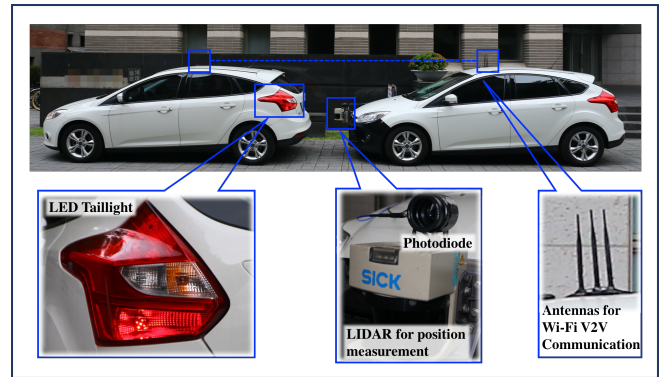

(b) System setup.

Figure 4: The V2V-VLC system used for the tests in real driving condition.

as shown on Figure 4(b). This system, when used with OFDM modulation, has already been described in detail in [9]. However, its overall functioning is reminded here.

On the transmitting side (i.e., the LV side), the V2VVLC system operates as follows:

- The data stream is first generated and encoded according to one of the modulations previously described using MATLAB on a laptop.

- The resulting data packet is uploaded to a universal software radio peripheral (USRP) Ettus N200 that turns it into a voltage signal and transmits it ad infinitum so that a continuous data stream is obtained.

- This voltage signal is fed to an LED driving circuit that converts it into a current signal adapted to the taillights requirements.

- The LED taillights of the LV, which are both controlled by the same LED driver, finally turn the driving current into the optical data signal of interest. Note that each taillight has a luminous in-



Figure 5: The optical system placed in front of the Thorlabs PDA100A to enhance the optical power received.

tensity in the reference axis of $9 \mathrm{~cd}$ and is fixed at $87 \mathrm{~cm}$ above ground.

On the receiving side (i.e., the FV side), the following operations are performed:

- The optical signals transmitted by the LV taillights are first received by a Thorlabs PDA100A photoreceiver, mounted on the front bumper of the FV. To obtain higher received power, an optical system is proposed in front of the PD, as illustrated by Figure 5. This optical system is composed first of an aspherical condenser lens of diameter $50 \mathrm{~mm}$ and focal length $32 \mathrm{~mm}$, placed in an adjustable collimation adapter. The back focal length of this lens is $17 \mathrm{~mm}$ which is smaller than the shortest length supported by the adjustable collimation adapter. Consequently, a plano-convex spherical lens of 50 $\mathrm{mm}$ focal length is added in order to make sure all the light collected by the condenser is projected on the sensitive area of the PD. This optical system allows to increase the light power collected by 15 $\mathrm{dB}$ and thus to increase the SNR, while limiting the FOV to $18^{\circ}$.

- The voltage signal output by the PD is then directly sampled by a second USRP Ettus N200 at a sampling rate depending on the modulation used, as explained in Section 2.1. Note that with OFDM, a $0.1 \mathrm{MHz}-8 \mathrm{GHz}$ analog bandpass filter is inserted between the PD and the USRP to cut the ambient light contribution. In any case, the samples are stored directly in a bin file on a laptop connected to the USRP.

- These samples are finally processed offline with MATLAB in order to decode the data received.

The various parameters of the emitter and receiver are summed up in Table 1. In order to investigate the impact of the relative positions of both vehicles on the 
data transmission performances, the $\mathrm{FV}$ is also equipped with a SICK LMS-291 LIDAR to measure both the longitudinal and lateral V2V distances. This LIDAR has an angular resolution of $0.25^{\circ}$ over a $100^{\circ} \mathrm{FOV}$ and a refresh rate of $37.5 \mathrm{~Hz}$. In addition, a Mio Combo 5107 embedding a global positioning system (GPS) and a camera is fixed on the windshield of the FV in order to record the global location of the cars and a real view of the driving conditions. Both vehicles, and more precisely their embedded laptops, are synchronized using the network time protocol (NTP). A NTP server is configured on the laptop of the FV and uses the GPS as time reference. Synchronization of the other vehicles is then achieved through a $5 \mathrm{GHz}$ WiFi connection based on ASUS RT-N66U wireless routers. Eventually, all the data collected by the laptops in both vehicles come with a timestamp, so that several time- and position-based analysis of the data transmission performances can be carried out, which will be presented in the next section.

\section{Results}

We have carried out measurements with the set-up introduced in Section 2 in real-world driving scenarios on a freeway. The experimental protocol followed during these tests is first explained. After verifying the different LED input current range of both modulations introduces similar output intensity, the performance comparison between OFDM and OOK is then presented. We detail the error rate performances of the OOK modulation and compare them with those of OFDM. The sensitivity to external interferences of both modulations is then studied.

\subsection{Experimental Protocol}

During our experiments, the two cars were manually driven in a car following setting along a predefined freeway segment of $18 \mathrm{~km}$ in Taiwan. This track, shown on Figure 6/a), was composed of uphill and downhill slopes, curves, tunnels and on/off ramps with sharp turns. The weather was mostly overcast, as shown on Figure 6(b), but with punctual peaks of light. Note, however, that the resulting daylight level was not recorded. In addition, the sun was never in direct lineof-sight (LOS) thanks to the restricted FOV of the receiver. Finally, in order to cover as much relative positions as possible, the driver of the receiving vehicle was asked to regularly change its longitudinal and lateral V2V positions. In the OOK configuration, the route was driven four times to increase the amount of data collected so that, eventually, around 45 minutes of recording were stored and exploited, corresponding to over

\begin{tabular}{|c|c|c|}
\hline Parameter & \multicolumn{2}{|c|}{ Value } \\
\hline $\begin{array}{l}\text { Transmitter } \\
\text { (LV) }\end{array}$ & OFDM & OOK \\
\hline $\begin{array}{c}\text { Number of } \\
\text { carriers }\end{array}$ & 16 & 1 \\
\hline $\begin{array}{c}\text { Carrier } \\
\text { Frequency }\end{array}$ & $200-400 \mathrm{kHz}$ & $200 \mathrm{kHz}$ \\
\hline $\begin{array}{c}\text { USRP DAC } \\
\text { Sampling Rate }\end{array}$ & $200 \mathrm{kHz}$ & $10 \mathrm{MHz}$ \\
\hline $\begin{array}{l}\text { LED Luminous } \\
\text { Intensity }\end{array}$ & \multicolumn{2}{|c|}{$2 \times 9 \mathrm{~cd}$} \\
\hline Height of Lamp & \multicolumn{2}{|c|}{$0.87 \mathrm{~m}$} \\
\hline $\begin{array}{c}\text { Packet } \\
\text { Definition }\end{array}$ & $\begin{array}{l}40 \text { OFDM } \\
\text { symbols }\end{array}$ & $\begin{array}{c}\text { Preamble bits } \\
\text { '1111' + } 400 \\
\text { data bits }\end{array}$ \\
\hline Receiver (FV) & OFDM & OOK \\
\hline Optical Gain & \multicolumn{2}{|c|}{$15 \mathrm{~dB}$} \\
\hline Receiver FOV & \multicolumn{2}{|c|}{$18^{\circ}$} \\
\hline $\begin{array}{l}\text { Photodiode } \\
\text { Module }\end{array}$ & \multicolumn{2}{|c|}{ Thorlabs PDA100A } \\
\hline Detection Area & \multicolumn{2}{|c|}{$10 \mathrm{~mm} \times 10 \mathrm{~mm}$} \\
\hline Bandwidth & \multicolumn{2}{|c|}{$860 \mathrm{kHz}$ at $20 \mathrm{~dB}$ gain } \\
\hline Gain & \multicolumn{2}{|c|}{$0.75 \times 10^{4} \mathrm{~V} / \mathrm{A}$ at $20 \mathrm{~dB}$ setting } \\
\hline $\begin{array}{c}\text { Height of } \\
\text { Photodiode }\end{array}$ & \multicolumn{2}{|c|}{$0.87 \mathrm{~m}$} \\
\hline $\begin{array}{c}\text { Analog } \\
\text { Bandpass Filter }\end{array}$ & $\begin{array}{c}0.1 \mathrm{MHz}-8 \\
\mathrm{GHz}\end{array}$ & None \\
\hline $\begin{array}{c}\text { USRP ADC } \\
\text { Sampling Rate }\end{array}$ & $200 \mathrm{kHz}$ & $2 \mathrm{MHz}$ \\
\hline
\end{tabular}

Table 1: V2V-VLC System Parameters. 


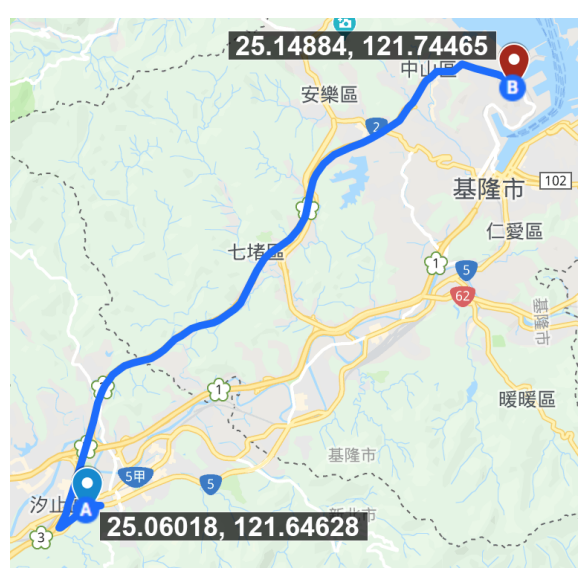

(a) A pre-defined $18 \mathrm{~km}$ driving route on Freeway No. 3 in Taiwan.



(b) Driving view captured by dashcam Mio Combo 5107 .

Figure 6: The real-world driving environment.

625 thousand packets (i.e., more than 250 million bits). As a reference, in the OFDM configuration, the route was driven for six times, collecting more than 15 thousand packets (i.e., more than 6 million bits). The large difference on received packet number is mainly caused by different packet duration used in the two modulation formats.

\subsection{Performance Comparison on Different Range of Input Current}

Before detailing the performance comparison between OFDM and OOK, we first check that the experiments are carried out on similar basis, especially regarding the LED output light intensity. In our prior work on OFDM, we downscaled the input current to only $9 \%$ of the original range to maintain the linearity required by OFDM. An LED is indeed a non-linear device, in the sense that the relationship between the input current and the output light intensity in a given direction is nonlinear. This non-linearity in an OFDM system would lead to undesired ISI. Therefore, the input current and the output intensity of an LED should be precisely calibrated.

On the other hand, in an OOK system, where the output intensity is only on and off, the impact of non-

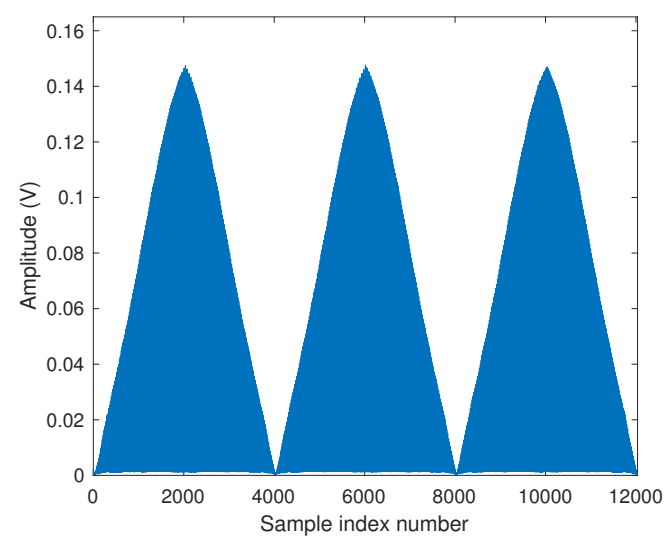

(a) OFDM with input current range equal to $9 \%$ of full range.

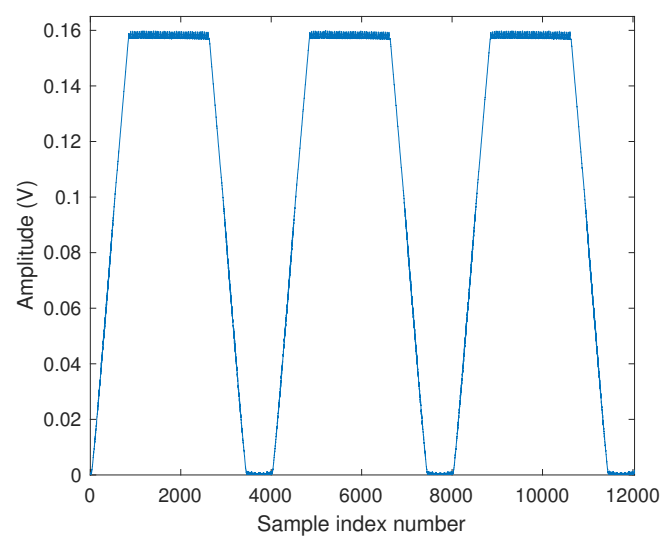

(b) OOK with input current range equal to the full range.

Figure 7: Comparison of the LED light intensity response to a triangle current command with OFDM and OOK

linearity is negligible. The full current range can thus be used. However, in such a case, the output light intensity in the receiver direction may be larger than with OFDM, since there is no current restriction. Such disparity would then influence the performance comparison that we want to carry out, and must thus be evaluated. Therefore, we further conduct experiments to verify how large the difference of the output intensity between the OFDM with linearity range and OOK with full range would be.

In our experiments, three different kinds of taillights are examined: the taillights of Ford Focus (the one being used in the next experiments), the taillight of Toyota Corolla, and the taillight of Mitsubishi Outlander. For OFDM, we use a triangle signal with a carrier frequency to include the maximal and minimal input current level in OFDM, and the input current is down-scaled to $9 \%$ of the original range. For OOK, the input current is the original range, and a triangle wave is also used to obtain 


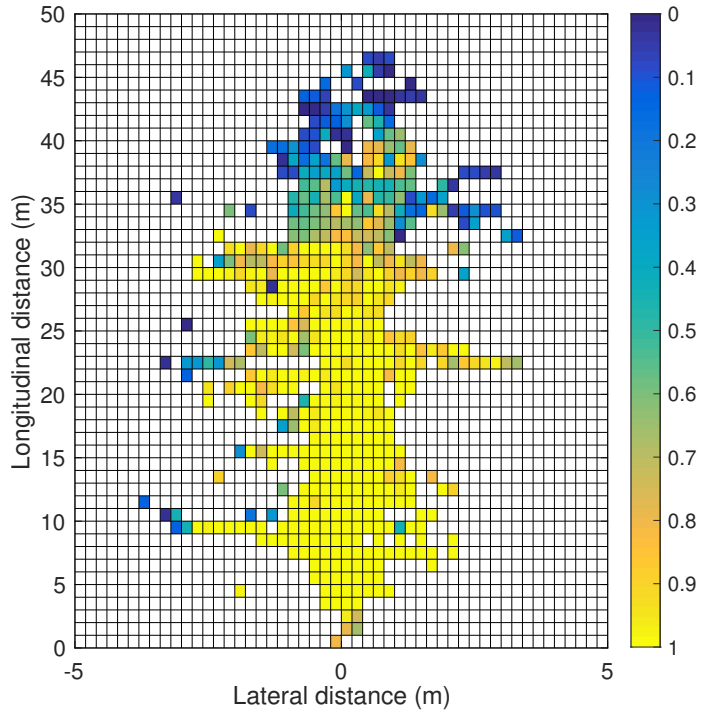

(a) PRR distribution of OOK at $100 \mathrm{kbps}$

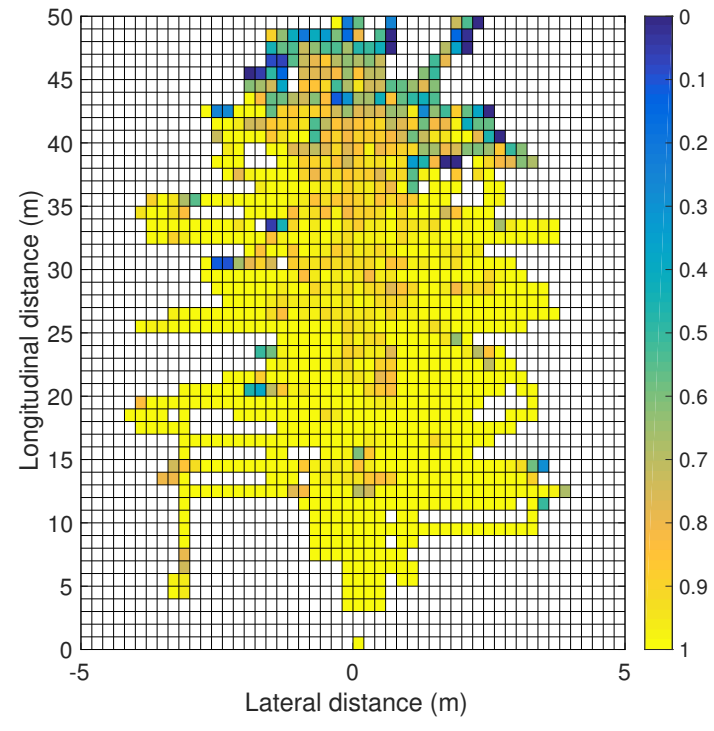

(b) SRR distribution of OFDM at $2 \mathrm{kbps}$.

Figure 8: Spatial distribution of the reception rate performance of OOK and OFDM.

the maxima and minima input current level. The LED output intensity is not measured directly but evaluated through a Thorlabs PDA100A photoreceiver, placed in the reference axis of the LED, and providing a voltage signal proportional to the incident light. The results obtained with the Ford Focus taillight are in Figure 7

As one can see, the maximal output intensity with OFDM $(0.15 \mathrm{~V})$ is very close to what OOK can achieve $(0.16 \mathrm{~V})$. In addition, the results obtained from the two other kinds of taillights are quite the same as the Focus taillight. This implies that when using OFDM as the modulation scheme, the down-scaling of input current will not decrease the output power level. Therefore, we can conclude that the results presented in the next subsection are based on LED taillights emitting the same light intensity in the receiver direction, regardless of the use of OFDM or OOK.

\subsection{Reception Rate Performances}

We can now compare the reception rate performances of OOK and OFDM. In OOK, this performance is evaluated through the spatial distribution of the PRR, that is the evolution of the PRR according to the longitudinal and lateral distance. The PRR is defined as the ratio between the number of packets received without any errors over the total number of transmitted packets. On the other hand, as indicated in Section 3.1, the number of OFDM packets received is about 40 times smaller than that of OOK. To maintain sufficient samples in one unit position block, we refer to the spatial distribution of the symbol reception rate (SRR) as the reception rate performance. The SRR is similarly defined as the ratio between the number of symbols received without any errors over the total number of transmitted symbols.

Figure 8 shows the resulting PRR and SRR spatial distribution of OOK and OFDM, respectively. The origin of each graph represents the position of the FV whereas the points on the grids are the various positions of the LV. The longitudinal and lateral resolutions of this mapping are respectively $1 \mathrm{~m}$ and $0.2 \mathrm{~m}$. The PRR and SRR in each $1 \times 0.2 \mathrm{~m}$ square are calculated over at least 5000 bits.

We can clearly see that with OOK, the reception rate remains over $90 \%$ up to around $30 \mathrm{~m}$ and then decreases dramatically. On the other hand, we can see with OFDM that the reception rate remains larger than $90 \%$ up to $35 \mathrm{~m}$, which confirms it has better performances than OOK, as shown in Section 2.2 The reception rate of OFDM also remains close to $100 \%$ for lateral shift of $\pm 4 \mathrm{~m}$. Such a lateral distance is actually larger than the typical width of a single highway lane, which is around $3.65 \mathrm{~m}$. Note that with OOK, the system supports lateral shifts of at least $\pm 1 \mathrm{~m}$ over around $30 \mathrm{~m}$, but there is not enough lateral data collected to conclude on its robustness to lateral shifts above $\pm 1 \mathrm{~m}$. However, as explained in Section 2.2, the error rate depends on the SNR. Thus, 


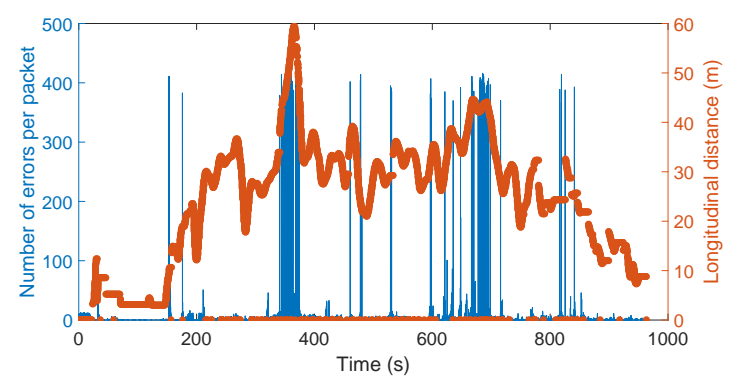

(a) Repetition factor $=1\left(R_{b}=100 \mathrm{kbps}\right)$.

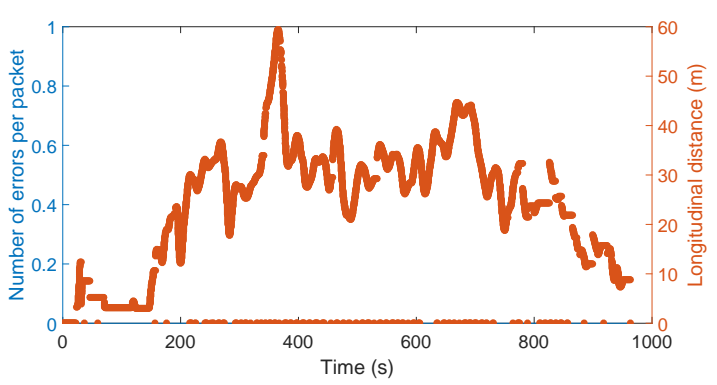

(b) Repetition factor $=10\left(R_{b}=10 \mathrm{kbps}\right)$.

Figure 9: Time evolution of the number of incorrect bits per packets without and with repetition coding.

when given a fixed noise level, the error rate would be highly correlated to the received optical power $P_{r}$, which is equal to the transmitted optical power $P_{t}$ attenuated by the channel DC gain $H(0)$. Figure 2 shows that $H(0)$ at close longitudinal distances but large lateral shifts has similar values as at larger longitudinal distances and zero lateral shift. More precisely, if the two vehicles are separated by an absolute distance of $d$ with a lateral shift corresponding to non-zero irradiance and incidence angles $\phi$ and $\psi$, then we can consider, with reference to Equation (2), that the $\cos ^{m} \phi \cos \psi$ penalty introduced by this lateral shift is equivalent to a virtual absolute distance $d^{\prime}$, where $d^{\prime}=\sqrt{d^{2} / \cos ^{m} \phi \cos \psi}>d$. Therefore, we can assume that OOK has a similar behavior as OFDM with large lateral shifts, although its maximum error-free lateral shift would be smaller than with OFDM.

From these results, we can conclude that our set-up has a range of operation that might seem limited for applications requiring long transmission distances. However, we can also conclude that VLC is definitely suited for medium-range applications like highway platooning since the longitudinal range of error-free communication is way larger than the optimal V2V distance required for such applications, defined as a $0.3 \mathrm{~s}$ gap corresponding to around $11 \mathrm{~m}$ at $130 \mathrm{~km} / \mathrm{h}$ [4]. The lateral

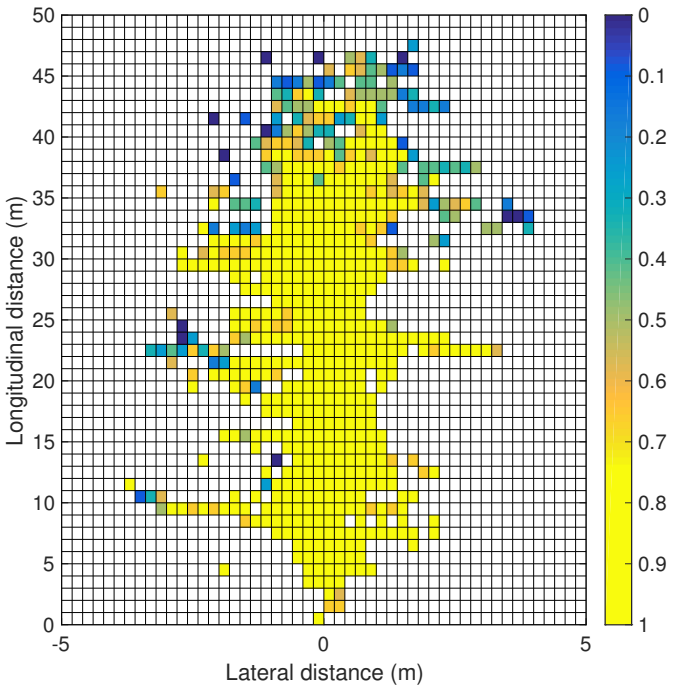

Figure 10: Spatial distribution of the PRR using OOK with a repetition coding factor is of $10\left(R_{b}=10 \mathrm{kbps}\right)$.

robustness is also such that most $\mathrm{V} 2 \mathrm{~V}$ relative positions occurring on highway platoons are supported.

However, these results also show some disparities between OFDM and OOK. Figure 9 a) shows the time evolution of the number of incorrect bits per packets and the V2V longitudinal distance when using OOK. On the one hand, it appears that even at low distance, there might be some occasional errors, even though their number remains rather low. This phenomenon has not been observed with OFDM. On the other hand, the number of incorrect bits soars up to almost 400, corresponding to the size of the entire packet, when the V2V distance is larger than around $30 \mathrm{~m}$. This latter effect, which confirms the quick collapse of the PRR observed on Figure 8 a), is not as strong with OFDM.

These differences can be mainly explained by the fact that with OFDM, a repetition coding scheme is used whereas it is not the case with OOK. Each symbol is indeed transmitted successively 50 times to improve the robustness of the link to SNR. However, with OOK, a single packet is transmitted continuously which means repetition coding can be implemented during the postprocessing phase. Figure 9 (b) shows that when the transmitted packet is repeated 10 times before decoding, the number of incorrect bits per packet decoded remains null whatever the V2V longitudinal distance. Figure 10 confirms this result by showing the spatial distribution of the PRR when a repetition factor of 10 is used. The PRR remains closer to 1 over a larger service area than 
without repetition coding. In particular, the maximum 'error-free' range reaches $35 \mathrm{~m}$, rather than $30 \mathrm{~m}$ previously. Consequently, as with OFDM, repetition coding increases the VLC transmission performances, but at the cost of the data rate, which drops to $10 \mathrm{kbps}$ here. On the other hand, despite that a repetition factor of 10 is adopted in OOK, the reception rate of OFDM over $40 \mathrm{~m}$ is still higher than that of OOK, due to the larger repetition factor used in OFDM. These experimental results are also in line with the simulation results shown in Figure 3 .

\subsection{Robustness to Interferences}

As already pointed out in [9] with OFDM, factors other than the V2V distance may cause reception errors. For instance, the interferences caused by infrastructure lighting were found to have a non-negligible impact. The highway track driven during the experiments includes tunnels equipped with several rows of three LED signs for lane indication, as shown on Figure 11.a). The frequency response of these signs, obtained by fast Fourier transform (FFT) of the light signal collected by the PD, is represented on Figure 11 (b). It exhibits narrow peaks at multiple of the fundamental frequency $135.025 \mathrm{kHz}$ and extends up to almost 700 $\mathrm{kHz}$.

As observed in [9], these interferences are narrowband enough to impact only a limited number of OFDM subcarriers. Figure 12 shows the bit error rate of each subcarrier while encountering the LED signage. As one can see that there is an increased error rate at frequency band from $250 \mathrm{kHz}$ to $290 \mathrm{kHz}$, which corresponds to frequency response observed in Figure 11(b). Even if the bits conveyed by the jammed subcarriers will be lost, the complete symbol can still be reconstructed without errors by using efficient forward error correction (FEC). Although not implemented here, such strategy is known to significantly improve the communication performances, even with strong interferences [15].

On the other hand, the Manchester modulation do not cope as well with these interferences. Here, the fundamental interference at $135.025 \mathrm{kHz}$ falls right in the middle of the main lobe of the $100 \mathrm{kbps}$ Manchester data signal. Consequently, it cannot be filtered out without removing at the same time a large part of the data signal power and thus lowering the SNR too much. In addition, the power of the interfering signal is way larger than that of the data signal. The spectrum on Figure 11(b) actually also includes the spectrum of the Manchester signal received but it is too low to be clearly visible. Consequently, the bit-error rate (BER) increases brutally when approaching an LED sign, as



(a) LED sign in the tunnel.

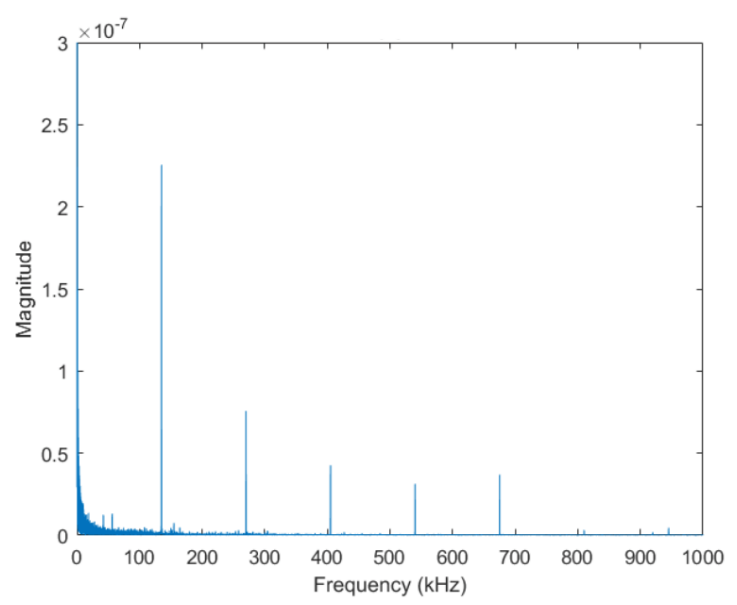

(b) FFT of the collected light signal of the LED signs.

Figure 11: Characterization of the LED signs encountered in the tunnels.

shown by Figure 13 . The VLC link thus becomes temporarily non-usable.

These results show that OFDM has a clear advantage over Manchester modulation, and SCM in general, to deal with external interferences. In addition, OFDM is known to be less sensitive to ISI than SCM. Here, the carrier frequencies used in both cases $-400 \mathrm{kHz}$ at most with OFDM and $200 \mathrm{kHz}$ with Manchester - are too low to cause ISI that would degrade the performance. The delay spread is indeed typically of a few tens of nanoseconds in $\mathrm{V} 2 \mathrm{~V}$ configurations, so the coherence bandwidth is usually larger than $10 \mathrm{MHz}$ [16]. However, if larger data rate and thus higher signal bandwidth are needed, OFDM would be preferred due to its ability to mitigate ISI. With Manchester modulation, the data rate is indeed half the carrier frequency $R_{b}=f_{c} / 2$ so ISI would start to appear from data rates around $5 \mathrm{Mbps}$. 


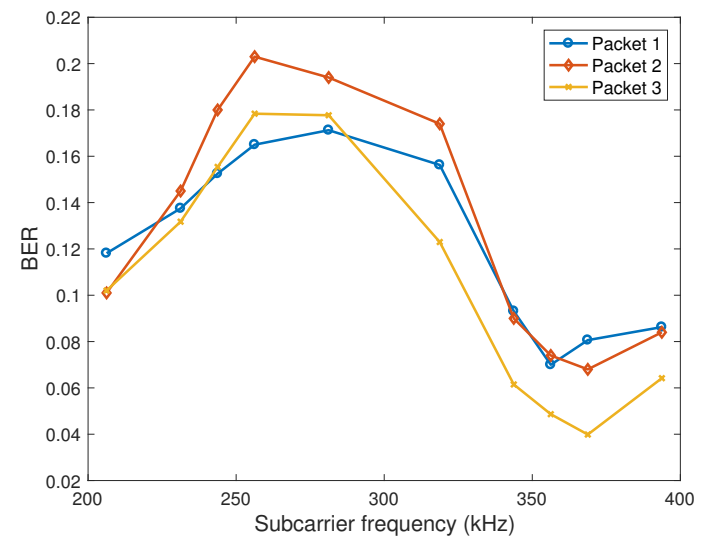

Figure 12: BER distribution over the OFDM subcarriers when encountering the LED signage.

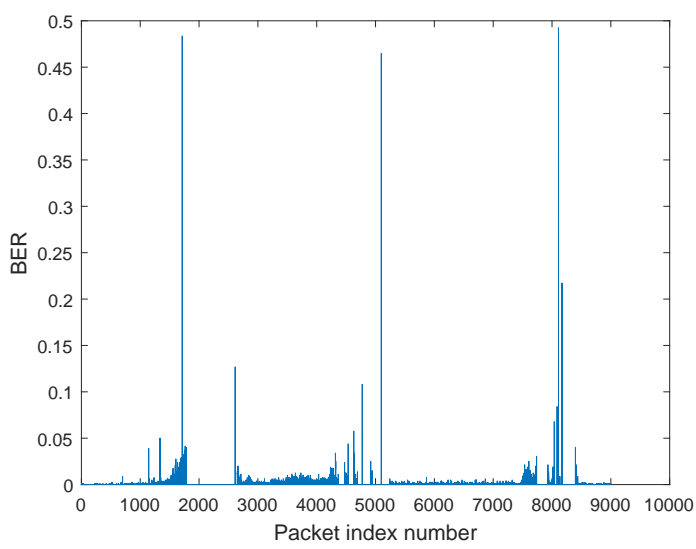

Figure 13: Evolution of the BER of the consecutive packets received with OOK when encountering the LED signage.

\section{Conclusions and Future Works}

In this work, we carry out real-world experiments with V2V-VLC using an SCM scheme. Along with the results reported in our previous work [9], performance comparisons between VLC using an SCM (e.g., OOK) and a MCM (e.g., OFDM) are introduced. The most significant implication in our finding is that we confirm that VLC is a promising candidate for V2V communications. The reception rate performance of OOK and OFDM both presents a reliable communication link for over $35 \mathrm{~m}$, regardless of the relatively lateral shift of \pm 1 m. In particular, a $100 \mathrm{kbps}$ data link is achieved with very light protocols. To the authors' knowledge, this paper, along with [9], is the first demonstration of a V2VVLC link on open roads with such rate and range performances. These results also provide strong supports for the suitability of VLC for short-range applications such as highway platooning.

In addition, the comparisons between OOK and OFDM modulations bring precious indications on the strength and limitations of both schemes. First, OFDM can only provide a data rate of $2 \mathrm{kbps}$, while OOK can have a much higher data rate of 10 or $100 \mathrm{kbps}$. This is mainly caused by the repetition coding used in OFDM to fight the power dispersion on multiple carriers. Second, OFDM exhibits a better resilience to external interferences than OOK. It is shown with OOK that LED signs can completely blind the receiver so that the link is temporarily non-usable, whereas with OFDM, some bits can still be received. In this way, it is possible to apply an efficient FEC to reconstruct the complete symbol. In parallel, we also show that the down-scaling of the input current for non-linearity problem in an OFDM system will not lead to significant difference on the output intensity compared to an OOK system using a full range of input current.

In conclusion, a higher data rate can be obtained using OOK while better interference resilience is shown when using OFDM. Though OOK could be modulated on a higher carrier frequency to avoid the external interferences, this solution is limited by the bandwidth of commodity LED, which usually does not exceed a few megahertz. Therefore, considering the varied driving environments, OFDM still remains the most serious solution, even though its data rate performances should be increased to support a wider range of applications. Therefore, future works will consist in improving the OFDM-based V2V-VLC link in terms of range and rate performances.

\section{Acknowledgment}

This research was supported in part by the Ministry of Science and Technology of Taiwan (MOST 1072633-E-002-001), National Taiwan University (NTU107L104039), Intel Corporation, and Delta Electronics.

\section{References}

\section{References}

[1] A. Festag, Cooperative intelligent transport systems standards in europe, IEEE Communications Magazine 52 (12) (2014) 166172. doi:10.1109/MCOM.2014.6979970

[2] A. Böhm, M. Jonsson, E. Uhlemann, Performance comparison of a platooning application using the IEEE 802.11p MAC on the control channel and a centralized MAC on a service channel, in: 2013 IEEE 9th International Conference on Wireless and Mobile Computing, Networking and Communications (WiMob), 2013, pp. 545-552. doi:10.1109/WiMOB. 2013.6673411 
[3] S. Tsugawa, S. Jeschke, S. E. Shladover, A Review of Truck Platooning Projects for Energy Savings, IEEE Transactions on Intelligent Vehicles 1 (1) (2016) 68-77. doi:10.1109/TIV. 2016.2577499

[4] European truck platooning network - vision truck platooning 2025

URL https://www.tno.nl/en/about-tno/news/ 2016/4/vision-truck-platooning-2025/

[5] A. Cailean, M. Dimian, Current Challenges for Visible Light Communications Usage in Vehicle Applications: A Survey, IEEE Communications Surveys Tutorials 19 (4) (2017) 26812703. doi:10.1109/COMST.2017.2706940

[6] T. Nagura, T. Yamazato, M. Katayama, T. Yendo, T. Fujii, H. Okada, Tracking an LED array transmitter for visible light communications in the driving situation, in: 2010 7th International Symposium on Wireless Communication Systems (ISWCS), 2010, pp. 765-769. doi:10.1109/ISWCS. 2010.5624361

[7] T. Yamazato, N. Kawagita, H. Okada, T. Fujii, T. Yendo, S. Arai, K. Kamakura, The Uplink Visible Light Communication Beacon System for Universal Traffic Management, IEEE Access 5 (2017) 22282-22290. doi:10.1109/ACCESS.2017. 2759179

[8] I. Takai, T. Harada, M. Andoh, K. Yasutomi, K. Kagawa, S. Kawahito, Optical Vehicle-to-Vehicle Communication System Using LED Transmitter and Camera Receiver, IEEE Photonics Journal 6 (5) (2014) 1-14. doi:10.1109/JPHOT. 2014.2352620

[9] W. Shen, H. Tsai, Testing vehicle-to-vehicle visible light communications in real-world driving scenarios, in: 2017 IEEE Vehicular Networking Conference (VNC), 2017, pp. 187-194. doi:10.1109/VNC.2017.8275596

[10] B. Béchadergue, L. Chassagne, H. Guan, Experimental comparison of pulse-amplitude and spatial modulations for vehicle-tovehicle visible light communication in platoon configurations, Optics Express 25 (20) (2017) 24790-24802. doi : 10 .1364/ OE.25.024790

[11] J. Kahn, J. Barry, Wireless infrared communications, Proceedings of the IEEE 85 (2) (1997) 265-298. doi:10.1109/5. 554222

[12] A. L. Chen, H. P. Wu, Y. L. Wei, H. M. Tsai, Time variation in vehicle-to-vehicle visible light communication channels, in: 2016 IEEE Vehicular Networking Conference (VNC), 2016, pp. 1-8. doi:10.1109/VNC.2016.7835926

[13] M. Uysal, Z. Ghassemlooy, A. Bekkali, A. Kadri, H. Menouar, Visible Light Communication for Vehicular Networking: Performance Study of a V2v System Using a Measured Headlamp Beam Pattern Model, IEEE Vehicular Technology Magazine 10 (4) (2015) 45-53. doi : 10 .1109/MVT . 2015.2481561

[14] Hua-Yen Tseng, Y. Wei, Ai-Ling Chen, Hao-Ping Wu, Hsuan Hsu, H. Tsai, Characterizing link asymmetry in vehicle-tovehicle visible light communications, in: 2015 IEEE Vehicular Networking Conference (VNC), 2015, pp. 88-95. doi: $10.1109 / \mathrm{VNC} .2015 .7385552$

[15] J. Armstrong, OFDM for Optical Communications, Journal of Lightwave Technology 27 (3) (2009) 189-204. doi:10. 1109 /JLT.2008.2010061

[16] S. Lee, J. K. Kwon, S.-Y. Jung, Y.-H. Kwon, Evaluation of visible light communication channel delay profiles for automotive applications, EURASIP Journal on Wireless Communications and Networking 2012 (1) (2012) 1-8. doi:10.1186/ 1687-1499-2012-370 